\title{
FIVE YEARS OF A YOUNG ADULT PSYCHIATRIC DAY HOSPITAL A PORTUGUESE EXPERIENCE
}

\author{
B. Martins Ramos ${ }^{1}$, F. Coutinho' ${ }^{1}$ E. Osório ${ }^{1,2}$, S. Timóteo ${ }^{1}$, I. Brandão ${ }^{1,2}$.
}

${ }^{1}$ Centro Hospitalar e Universitário de São João, Serviço de Psiquiatria e Saúde Mental da Clínica de Psiquiatria e Saúde Mental ${ }^{2}$ Faculdade de Medicina da Universidade do Porto, Departamento de Neurociências Clínicas e Saúde Mental, Porto, Portugal.

The program: Psychiatric Young Adult Day Hospital (YADH) of the Department of Psychiatry and Mental Health of Centro Hospitalar e Universitário de São João was created in 2014, proposing a model of partial hospitalization for youths between 18 and 25 years with mental disorders.

Regarding the different psychiatric diagnosis and its variable presentation in this age range, the target of this the program has its own specifities.

The day hospital has a capacity for 10 patients and works in a day schedule from 9am to $4 \mathrm{pm}$. The therapeutic team includes psychiatrists, psychologists, occupational therapists and nurses.

It is a suitable model for youths with mental illness which provides a intensive care, without removing the patient from his family and from the social or educative environment. The objectives of this program reside on intervening on multiple causes behavioral crisis, on promoting or consolidating the psychopathological stabilization and diagnosis clarification. The therapeutic plan can vary depending on the patient and the aims are defined to the patient. This means highlining the therapeutic adhesion in several cases, the psychotherapeutic process in others or an occupational plan in the remaining.

This particular setting provides some therapeutic resources, such as regular psychotherapy, both in individual or group setting and occupational therapy. Concerning these, there are psychodrama groups where patients engage as a part of the treatment plan. Additionally, the program delivers an important scenery for the families to get involved in the particularities of the illness and to be helped in dealing with the process, discovering ways which they can be helpful in the therapeutic process. For this matter, systemic and family therapy is a resource available when considered useful.

\section{Results:}

$\rightarrow 88$ patients were admitted in YADH, consisting in a total of 127 admissions. The average age was 23.6 years old

$\rightarrow$ The education level was higher on the female population. In total, $45 \%$ were students at the moment of the admission.

$\rightarrow$ The average length of stay was 103 days.

$\rightarrow 72 \%$ were referred from an outpatient psychiatric appointment, while the rest were referred from in-patient treatment.

$\rightarrow$ At the time of discharge, $73 \%$ were considered clinically improved, while $7 \%$ worsened and were admitted in a acute psychiatric ward.

$\rightarrow$ Regarding the diagnosis at time of discharge, Eating Disorders were the most prevalent (41\%) in female population. $48 \%$ of the male population presented a diagnosis included in the group of Schizophrenia, Schizotypal and Delusional disorders, according to ICD-10.

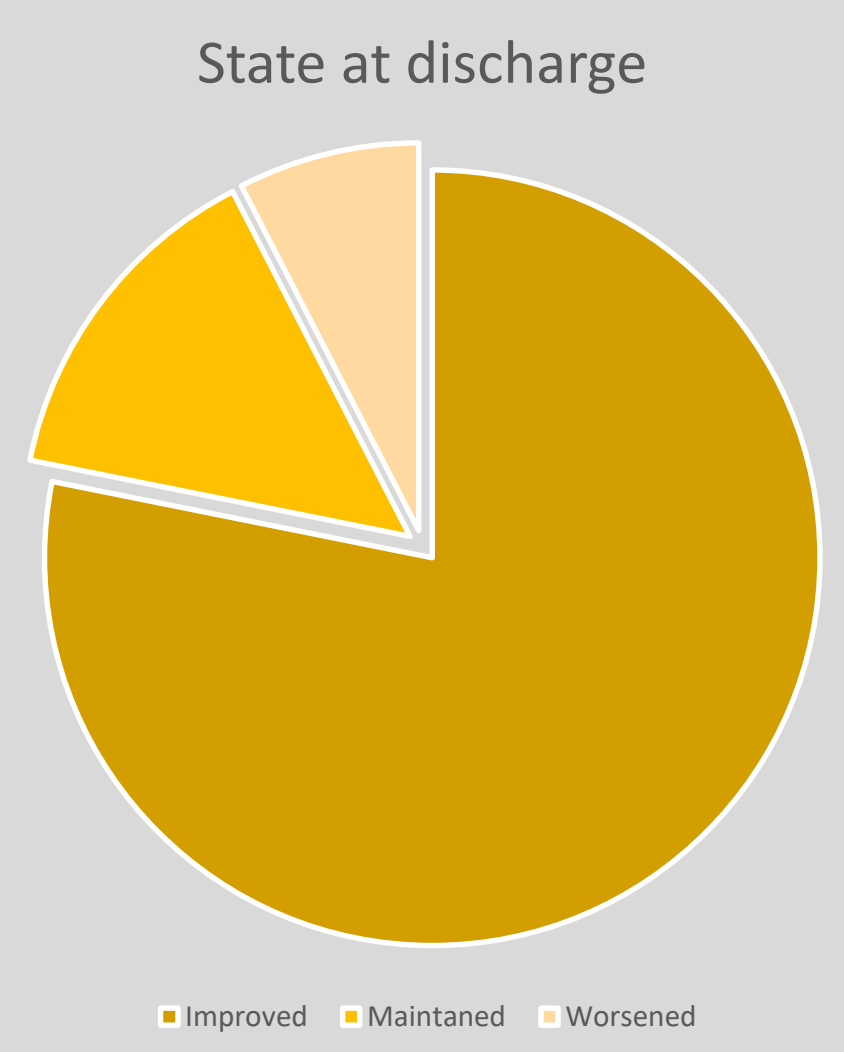

Patients' diagnosis at discharge

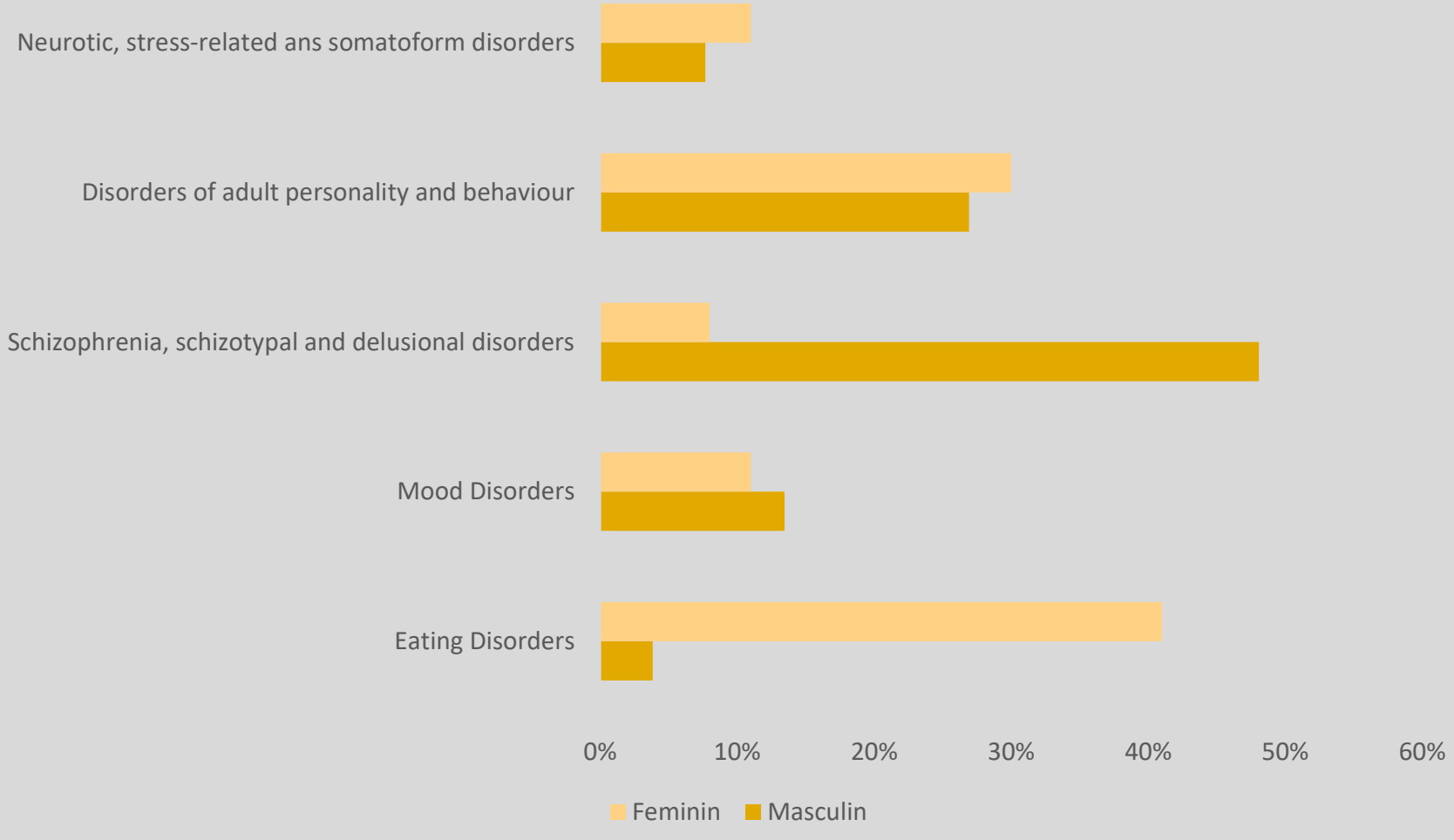

Conclusions: In this population there is a wide range of psychiatric diagnoses. Considering this, the preferential approach needs to be individualized, with the support of a multidisciplinary team.

Our model also proposes work with the patients' families, from a systemic perspective, while promoting the youths' autonomy. 